\title{
ON ARITHMETIC INVARIANTS OF BINARY CUBIC AND BINARY TRILINEAR FORMS*
}

\author{
BY RUFUS OLDENBURGER
}

1. Introduction. It is the purpose of this note to show that binary cubic and binary trilinear forms can be completely and very simply characterized by arithmetic rank invariants for nonsingular linear transformations in the complex field. We define the factorization rank $\nmid$ of a matrix $A=\left(a_{i j k}\right)$ of order $n$ and its associated trilinear form to be the minimum value of $\epsilon$ such that $A$ can be "factored" into the form

$$
A=\left(\sum_{\alpha=1}^{\epsilon} a_{\alpha i} b_{\alpha j} c_{\alpha k}\right), \quad(i, j, k=1,2, \cdots, n) .
$$

Hitchcock $\ddagger$ obtained minimum values of $\epsilon$ for certain polyadics. The number $\epsilon$ is invariant under non-singular linear transformations on the variables in the trilinear form

$$
\sum a_{i j k} x_{i} y_{j} z_{k}
$$

associated with $A$. In a paper which appeared in this Bulletin, $\S$ I classified binary trilinear forms by means of ranks and a property of invariant factors, which are invariant under non-singular linear transformations in the complex field. The ranks of that paper and the rank defined above form a complete invariant system for these forms. For binary cubic forms the factorization rank alone forms a complete invariant system.

2. Factorization Ranks. The canonical binary trilinear forms are

* Presented to the Society, April 11, 1936.

$\dagger$ This rank was used in developing a general theory of non-singular $p$-way matrices in the paper Non-singular multilinear forms and certain p-way matrix factorizations, Transactions of this Society, vol. 39 (1936), pp. 422-455. If factorization rank is similarly defined for 2 -way matrices, it is found that the factorization rank of a 2-way matrix is $n$ if and only if its ordinary rank is $n$.

$\ddagger$ F.L.Hitchcock, The expression of a tensor or polyadic as a sum of products, Journal of Mathematics and Physics, Massachusetts Institute of Technology, . vol. 6 (1927), pp. 164-189.

§ On canonical binary trilinear forms, vol. 38 (1932), pp. 385-387. 


$$
\begin{aligned}
R & =x_{1} y_{1} z_{1}+x_{2} y_{2} z_{2}, \\
L & =x_{1} y_{1} z_{1}+x_{2} y_{2} z_{1}+x_{2} y_{1} z_{2}, \\
H & =x_{1} y_{1} z_{1}+x_{1} y_{2} z_{2}, \\
K & =x_{1} y_{1} z_{1} .
\end{aligned}
$$

The forms $H, K$ can be distinguished from each other and from $L, R$ by the ranks of my Bulletin paper referred to above. Since the $i$-, $j$-, and $k$-ranks* of $L$ equal 2 , the factorization rank $\epsilon$ of $L$ is $\geqq 2$. If $\epsilon$ were equal to 2 for $L$, the form $L$ would be equivalent to $R$, which is impossible. For then the matrix $A$ of $L$ is of the form (1), where $A=\left(a_{\alpha i}\right), B=\left(b_{\alpha j}\right), C=\left(c_{\alpha k}\right)$ are non-singular, whence transformations on $L$ with $A^{-1}, B^{-1}, C^{-1}$ give $R$. Since the matrix of $L$ can be written in the form (1), where

$$
A=\left(\begin{array}{ll}
1 & 0 \\
0 & 1 \\
0 & 1
\end{array}\right), \quad B=\left(\begin{array}{ll}
1 & 0 \\
1 & 0 \\
0 & 1
\end{array}\right), \quad C=\left(\begin{array}{ll}
1 & 0 \\
0 & 1 \\
1 & 0
\end{array}\right),
$$

it follows that $\epsilon=3$.

The factorization rank $\epsilon$ for $H$ is $\geqq 2$, since the $j$ - and $k$-ranks of $H$ equal 2. That $\epsilon=2$ is evident since the matrix of $H$ can be written in the form (1), where

$$
A=\left(\begin{array}{ll}
1 & 0 \\
1 & 0
\end{array}\right), \quad B=C=\left(\begin{array}{ll}
1 & 0 \\
0 & 1
\end{array}\right) \text {. }
$$

The matrix of $K$ can be written in the form (1), where

$$
A=B=C=(1,0) \text {, }
$$

whence $\epsilon=1$.

The canonical binary cubic forms for the complex field are well known. $\dagger$ They are

$$
P=x^{3}+y^{3}, \quad Q=x^{2} y, \quad S=x^{3} .
$$

Since the matrix of $P$ can be taken to be the same as the matrix of $R$, its factorization rank is 2 . The matrix of $3 Q$ can be taken to be the matrix $A$ whose only non-vanishing elements are

* The $i$-rank of $A=\left(a_{i j k}\right),(i, j, k=1,2)$, is 2 if the minors $\left(a_{1 j k}\right)$ and $\left(a_{2 j k}\right)$ are linearly independent.

† L. E. Dickson, Modern Algebraic Theories, pp. 7-9. 
$a_{112}=a_{121}=a_{212}=1$. The trilinear form associated with this matrix is $x_{1} y_{1} z_{2}+x_{1} y_{2} z_{1}+x_{2} y_{1} z_{1}$, which is equivalent to $L$ under the transformations $x_{1}=x_{2}^{\prime}, x_{2}=x_{1}^{\prime}$. Since the matrices of $S$ and $K$ can be taken to be the same, the factorization rank of $S$ is 1 . We have proved the following result.

THEOREM. The factorization ranks of the forms in the sets $(K, S),(R, P, H)$, and $(L, Q)$ are 1,2 , and 3 , respectively.

The equivalence of cubics to $P, Q, S$ can be recognized very simply without the use of factorization rank from the theory of my previous Bulletin paper.

Armour Institute of Technology

\section{A NOTE ON THE DEGREE OF POLYNOMIAL APPROXIMATION*}

\section{BY J. H. CURTISS}

Let $C$ be a rectifiable Jordan curve of the finite $z$ plane. We shall say that a function $f(z)$ belongs to the class $\operatorname{Lip}(C, j, \alpha)$ if $f(z)$ is regular in the limited region bounded by $C$ (which we shall call the interior of $C$ ), if $f(z)$ is continuous in the corresponding closed region, and if the $j$ th derivative of $f(z)$ is also continuous in this closed region and satisfies a Lipschitz condition with exponent $\alpha$ on $C$ :

$$
\left|f^{(j)}\left(z_{1}\right)-f^{(j)}\left(z_{2}\right)\right| \leqq M\left|z_{1}-z_{2}\right|^{\alpha}
$$

$z_{1}, z_{2}$ on $C$. The number $\alpha$ will be positive and not greater than unity. The number $j$ will be a positive integer or zero; we define $f^{(0)}(z)$ to be identically $f(z)$. The object of this note is to establish the following existence theorem.

THEOREM. Let the point set $S$ consist of a finite number of closed limited Jordan regions of the z plane bounded by the mutually exterior analytic curves $C_{1}, C_{2}, \cdots, C_{\lambda}$. Let the functions $f_{1}(z), f_{2}(z), \cdots, f_{\lambda}(z)$ belong respectively to the classes

$$
\operatorname{Lip}\left(C_{1}, j, \alpha\right), \quad \operatorname{Lip}\left(C_{2}, j, \alpha\right), \quad \cdots, \quad \operatorname{Lip}\left(C_{\lambda}, j, \alpha\right),
$$

\footnotetext{
* Presented to the Society, December 31, 1935.
} 